\title{
Analytic solutions of linear difference equations, formal series, and bottom summation
}

\author{
S. A. Abramov ${ }^{1 \star}$ and M. Petkovšek ${ }^{2 \star \star}$ \\ 1 Russian Academy of Sciences, Dorodnicyn Computing Centre, \\ Vavilova 40, 119991, Moscow GSP-1, Russia (sabramov@ccas.ru) \\ ${ }^{2}$ University of Ljubljana, Faculty of Mathematics and Physics, \\ Jadranska 19, SI-1000 Ljubljana, Slovenia (marko.petkovsek@uni-lj.si)
}

\begin{abstract}
We consider summation of consecutive values $\varphi(v), \varphi(v+1)$, $\ldots, \varphi(w)$ of a meromorphic function $\varphi(z)$ where $v, w \in \mathbb{Z}$. We assume that $\varphi(z)$ satisfies a linear difference equation $L(y)=0$ with polynomial coefficients, and that a summing operator for $L$ exists (such an operator can be found - if it exists - by the Accurate Summation algorithm, or alternatively, by Gosper's algorithm when ord $L=1$ ).

The notion of bottom summation which covers the case where $\varphi(z)$ has poles in $\mathbb{Z}$ is introduced.
\end{abstract}

\section{Introduction}

Similarly to $[8,3,5,1]$, this paper is concerned with the problem of summing the elements of a $P$-recursive sequence $f(k), k \in \mathbb{Z}$, i.e., a sequence which satisfies a linear difference equation with polynomial coefficients.

Let $E_{k}$ be the shift operator such that $E_{k}(f(k))=f(k+1)$ for sequences $f(k)$ where $k \in \mathbb{Z}$. Let

$$
L=a_{d}(k) E_{k}^{d}+\cdots+a_{1}(k) E_{k}+a_{0}(k) \in \mathbb{C}(k)\left[E_{k}\right] .
$$

We say that an operator $R \in \mathbb{C}(n)\left[E_{k}\right]$ is a summing operator for $L$ if

$$
\left(E_{k}-1\right) \circ R=1+M \circ L
$$

for some $M \in \mathbb{C}(k)\left[E_{k}\right]$. It is easy to see that if there exists a summing operator for $L$, then there also exists one of order $<d$ (simply replace $R$ by its remainder when divided by $L$ from the right). Hence we can assume w.l.g. that ord $R=$ ord $L-1=d-1$ :

$$
R=r_{d-1}(k) E_{k}^{d-1}+\cdots+r_{1}(k) E_{k}+r_{0}(k) \in \mathbb{C}(k)\left[E_{k}\right] .
$$

If a summing operator exists, then it can be constructed by the Accurate Summation algorithm [3] or, when $d=1$, by Gosper's algorithm [8]. In those cases

\footnotetext{
^ Partially supported by RFBR under grant 07-01-00482-a.

** Partially supported by ARRS under grant P1-0294.
} 
where $R \in \mathbb{C}\left[k, E_{k}\right]$ exists, equality (2) gives an opportunity to use the discrete Newton-Leibniz formula

$$
\sum_{k=v}^{w-1} f(k)=g(w)-g(v)
$$

for all integers $v<w$, and for any sequence $f$ such that $L(f)=0$, taking $g=R(f)$.

However, it was shown in [5] that if $R$ has rational-function coefficients which have poles in $\mathbb{Z}$, then this formula may give an incorrect result (see Example 5 of the present paper). This gives rise to defects in many implementations of summation algorithms. In $[5,1]$ a way was proposed to construct a basis for the space $W_{L, R}$ of all solutions of $L(y)=0$ for which (4) is valid for all integers $v<w$. It was also proved that $\operatorname{dim} W_{L, R}>0$ in the case $d=1$.

In the present paper we give a new sufficient condition for the correctness of definite summation by Gosper's algorithm and by the Accurate Summation algorithm.

In Section 3 below we prove that if a summing operator exists for $L$ with ord $L=d$, then $\operatorname{dim} W_{L, R}>0$ regardless of the value of $d$.

In Section 4 we suppose that $L$ acts on analytic functions:

$$
L=a_{d}(z) E_{z}^{d}+\cdots+a_{1}(z) E_{z}+a_{0}(z) \in \mathbb{C}(z)\left[E_{z}\right],
$$

where $E_{z}(\varphi(z))=\varphi(z+1)$ for analytic functions $\varphi(z)$ where $z \in \mathbb{C}$. We consider the summing operator (if it exists) in the form

$$
R=r_{d-1}(z) E_{z}^{d-1}+\cdots+r_{1}(z) E_{z}+r_{0}(z) \in \mathbb{C}(z)\left[E_{z}\right] .
$$

Let $\varphi(z)$ be a meromorphic solution of $L(y)=0$. It turns out that if $\varphi(z)$ has no pole in $\mathbb{Z}$, then $R(\varphi)(z)$ has no pole in $\mathbb{Z}$ as well, and we can use (4) to sum values $\varphi(k)$ for $k=v, v+1, \ldots, w$. This follows from a stronger statement also proved in Section 4 . The fact is that even if $\varphi(z)$ has some poles in $\mathbb{Z}$, the summation task can nevertheless be performed correctly. For any $k \in \mathbb{Z}$ the function $\varphi(z)$ can be represented as

$$
\varphi(z)=c_{k, \rho_{k}}(z-k)^{\rho_{k}}+c_{k, \rho_{k}+1}(z-k)^{\rho_{k}+1}+\ldots
$$

with $\rho_{k} \in \mathbb{Z}$ and $c_{k, \rho_{k}} \neq 0$. If $L(\varphi)=0$, then there exists the minimal element $\rho$ in the set of all $\rho_{k}, k \in \mathbb{Z}$. We associate with $\varphi(z)$ the sequence $f(k)$ such that $f(k)=c_{k, \rho_{k}}$ if $\rho_{k}=\rho$, and $f(k)=0$ otherwise. Then the sequence $f(k)$ satisfies the equation $L(y)=0$, if we use $E_{k}$ instead of $E_{z}$ in $L$. We associate a sequence $g(k)$ with $R(\varphi)$ in a similar way, and the value of $\rho$ for $R(\varphi)$ will be the same as for $\varphi$. Now formula (4) is correct. This type of summation we call bottom summation.

Some important auxillary statements (Section 2) on sequences of power series are based on the idea of the $\varepsilon$-deformation of a difference operator which was first used by M. van Hoeij in [7]; later this idea was used in [4] and in [2] as well. 


\section{Series-Valued Sequences}

We start with some notations and definitions. Let $\varepsilon$ be a variable (rather than a "small number"). As usual, $\mathbb{C}[[\varepsilon]]$ is the ring of formal power series in $\varepsilon$ and $\mathbb{C}((\varepsilon))=\mathbb{C}[[\varepsilon]]\left[\varepsilon^{-1}\right]$ is its quotient field (the field of formal Laurent series in $\varepsilon$ ).

If $s \in \mathbb{C}((\varepsilon)) \backslash\{0\}$ then we define the valuation of $s$ in the following way:

$$
\nu(s)=-\min \left\{m \mid m \in \mathbb{Z}, \varepsilon^{m} s \in \mathbb{C}[[\varepsilon]]\right\},
$$

in addition we set $\nu(0)=\infty$. If $s \in \mathbb{C}((\varepsilon)), m \in \mathbb{Z}$ then $\left[\varepsilon^{m}\right] s$ is the coefficient of $\varepsilon^{m}$ in the series $s$, and $\left[\varepsilon^{\infty}\right] 0=0$. It follows from the definition of the valuation that if $s, t \in \mathbb{C}((\varepsilon))$ then

$$
\nu(s t)=\nu(s)+\nu(t), \quad\left[\varepsilon^{\nu(s t)}\right](s t)=\left([ ( \varepsilon ^ { \nu ( s ) } ] s ) \left(\left[\left(\varepsilon^{\nu(t)}\right] t\right),\right.\right.
$$

and

$$
\nu(s+t) \geq \min \{\nu(s), \nu(t)\} .
$$

If $K$ is a ring, then $K^{\mathbb{Z}}$ denotes the ring of all maps $\mathbb{Z} \rightarrow K$, i.e., the ring of all two-sided $K$-valued sequences. Note that the operator $E_{k}$ is a ring automorphism of $K^{\mathbb{Z}}$.

If $S \in \mathbb{C}((\varepsilon))^{\mathbb{Z}}$, then $\nu(S)$ denotes the sequence in $\mathbb{Z}^{\mathbb{Z}}$ whose $k$-th element is $\nu(S(k))$. If $m \in \mathbb{Z}$, then $\left[\varepsilon^{m}\right] S$ denotes the sequence in $\mathbb{C}^{\mathbb{Z}}$ whose $k$-th element is $\left[\varepsilon^{m}\right](S(k))$. We say that $S$ is of bounded depth if the sequence $\nu(S)$ is bounded from below, i.e., there exists

$$
m=\min _{k} \nu(S(k)) .
$$

If $S$ is of bounded depth, then $m$ in (8) is the depth of $S$. In this case the bottom of $S$, which is a sequence in $\mathbb{C}^{\mathbb{Z}}$, is defined by

$$
\operatorname{bott}(S)=\left[\varepsilon^{m}\right] S .
$$

An operator $\Lambda \in \mathbb{C}((\varepsilon))^{\mathbb{Z}}\left[E_{k}\right]$ of the form

$$
\Lambda=S_{d} E_{k}^{d}+\cdots+S_{1} E_{k}+S_{0}, \quad S_{0}, S_{1}, \ldots, S_{d} \in \mathbb{C}((\varepsilon))^{\mathbb{Z}},
$$

defines a map $\mathbb{C}((\varepsilon))^{\mathbb{Z}} \rightarrow \mathbb{C}((\varepsilon))^{\mathbb{Z}}$ where $(\Lambda S)(k)=\sum_{j=0}^{d} S_{j}(k) S(k+j)$. If each sequence $S_{j}$ has bounded depth $m_{j}$ for $j=0,1, \ldots, d$, then we say that $\Lambda$ is of bounded depth $m=\min _{0 \leq j \leq d} m_{j}$. In this case the bottom of $\Lambda$ is

$$
\operatorname{bott}(\Lambda)=\sum_{j=0}^{d}\left(\left[\varepsilon^{m}\right] S_{j}\right) E_{k}^{j} \in \mathbb{C}^{\mathbb{Z}}\left[E_{k}\right] .
$$

Proposition 1. Let $\Lambda$ be an operator of the form (9), of bounded depth. Let $S \in \mathbb{C}((\varepsilon))$ satisfy $\Lambda(S)=0$. If for all but finitely many $k \in \mathbb{Z}$ we have

$$
\nu\left(S_{0}(k)\right)=\nu\left(S_{d}(k)\right)=\min _{0 \leq j \leq d} \nu\left(S_{j}(k)\right),
$$

then $S$ is of bounded depth and $\tilde{\Lambda}(\operatorname{bott}(S))=0$, where $\tilde{\Lambda}=\operatorname{bott}(\Lambda)$. 
Proof. Fix $k \in \mathbb{Z}$ and $i \in\{0,1, \ldots, d\}$. From $\Lambda(S)=0$ it follows that

$$
\nu\left(S_{i}(k) S(k+i)\right)=\nu\left(-\sum_{0 \leq j \leq d, j \neq i} S_{j}(k) S(k+j)\right),
$$

so by (6) and (7) we have

$$
\nu\left(S_{i}(k)\right)+\nu(S(k+i)) \geq \min _{\substack{0 \leq j \leq d \\ j \neq i}} \nu\left(S_{j}(k)\right)+\min _{\substack{0 \leq j \leq d \\ j \neq i}} \nu(S(k+j)) .
$$

Assume that $\nu\left(S_{i}(k)\right)=\min _{0 \leq j \leq d} \nu\left(S_{j}(k)\right)$. Then it follows from (11) that $\nu(S(k+i)) \geq \min _{\substack{0 \leq j \leq d \\ j \neq i}} \nu(S(k+j))$. Specializing this to $i=0$ and $i=d$ and using (10) we obtain that

$$
\nu(S(k)) \geq \min _{1 \leq j \leq d} \nu(S(k+j))
$$

and

$$
\nu(S(k+d)) \geq \min _{0 \leq j \leq d-1} \nu(S(k+j))
$$

for all but finitely many $k \in \mathbb{Z}$. Therefore $S$ is of bounded depth. The equality $\tilde{\Lambda}(\operatorname{bott}(S))=0$ now follows from $(6)$.

Example 1. Let

$$
\Lambda=S_{1} E_{k}+S_{0}, S_{1}(k)=k+1+\varepsilon, S_{0}(k)=-k-\varepsilon
$$

and

$$
S(k)= \begin{cases}-\frac{1}{\varepsilon}, & \text { if } k=0, \\ \sum_{i=0}^{\infty}\left(-\frac{1}{k}\right)^{i+1} \varepsilon^{i}, \text { otherwise. }\end{cases}
$$

Then $S_{1}(k) S(k+1)=-S_{0}(k) S(k)=-1$ for all $k$, and $\Lambda(S)=0$ as a consequence. The depth of $S$ is -1 .

We see that

$$
\operatorname{bott}(S)(k)= \begin{cases}-1, & \text { if } k=0, \\ 0, & \text { otherwise, }\end{cases}
$$

and $\operatorname{bott}(\Lambda)=(k+1) E_{k}-k$. It is easy to see that $(k+1) f(k+1)-k f(k)=0$, where $f(k)=\operatorname{bott}(S)(k)$; so $\tilde{\Lambda}(\operatorname{bott}(S))=0$, where $\tilde{\Lambda}=\operatorname{bott}(\Lambda)$.

\section{When a Summing Operator Exists}

If $\varphi(z) \in \mathbb{C}(z)$, then we write $\hat{\varphi}(k)$ for the sequence $\varphi(k+\varepsilon), k \in \mathbb{Z}$, of rational functions expanded into Laurent series about $\varepsilon=0$. We associate with every operator

$$
N=b_{l}(z) E_{z}^{l}+\cdots+b_{1} E_{z}+b_{0}(z) \in \mathbb{C}(z)\left[E_{z}\right]
$$

the operator

$$
\hat{N}=\hat{b}_{l}(k) E_{k}^{l}+\cdots+\hat{b}_{1}(k) E_{k}+\hat{b}_{0}(k) \quad \in \mathbb{C}((\varepsilon))^{\mathbb{Z}}\left[E_{k}\right]
$$

which acts on sequences from $\mathbb{C}((\varepsilon))^{\mathbb{Z}}$. 
Proposition 2. Let $L \in \mathbb{C}\left[z, E_{z}\right]$. Assume that $R \in \mathbb{C}(z)\left[E_{z}\right]$ is a summing operator for L. Let $S \in \mathbb{C}((\varepsilon))^{\mathbb{Z}}$ be such that $\hat{L}(S)=0$. Then

$$
\left(E_{k}-1\right)(\hat{R}(S))=S .
$$

Proof. By (2), there is an operator $M \in \mathbb{C}(z)\left[E_{z}\right]$ such that

$$
\left(E_{z}-1\right) \circ R=1+M \circ L .
$$

The map $N \mapsto \hat{N}$ is a ring homomorphism from $\mathbb{C}(z)\left[E_{z}\right]$ to $\mathbb{C}((\varepsilon))^{\mathbb{Z}}\left[E_{k}\right]$. Therefore (13) implies

$$
\left(E_{k}-1\right) \circ \hat{R}=1+\hat{M} \circ \hat{L} .
$$

Applying both sides of this equality to $S$, we obtain (12).

Proposition 3. Let $L \in \mathbb{C}\left[z, E_{z}\right]$, and let $R \in \mathbb{C}(z)\left[E_{z}\right]$ be a summing operator for L. Let $S \in \mathbb{C}((\varepsilon))^{\mathbb{Z}}$ be such that $\hat{L}(S)=0$. Then $\operatorname{depth}(\hat{R}(S))=\operatorname{depth}(S)$, and

$$
\left(E_{k}-1\right)(\operatorname{bott}(\hat{R}(S)))=\operatorname{bott}(S) .
$$

Proof. It follows from $(12)$ that $\operatorname{depth}(\hat{R}(S)) \leq \operatorname{depth}(S)$. To prove equality, we distinguish two cases.

1. $\operatorname{depth}(\hat{R}(S))=\nu(\hat{R}(S)(k))$ for all $k \in \mathbb{Z}$.

Assume that $\operatorname{depth}(\hat{R}(S))<\operatorname{depth}(S)$. Then $\operatorname{bott}(\hat{R}(S))$ is a non-zero constant sequence. However since $R$ has rational coefficients, there exists $k_{0} \in \mathbb{Z}$ such that for all $k \geq k_{0}$, the valuation of any coefficient of $\hat{R}$ is non-negative and, as a consequence,

$$
\nu(\hat{R}(S)(k)) \geq \min _{0 \leq i \leq \text { ord } R} \nu(S(k+i)) \geq \operatorname{depth}(S)>\operatorname{depth}(\hat{R}(S))
$$

for all $k \geq k_{0}$. Then $\operatorname{bott}(\hat{R}(S))(k)=0$ for all $k \geq k_{0}$. Hence $\operatorname{bott}(\hat{R}(S))$ is not a non-zero constant sequence. This contradiction implies that $\operatorname{depth}(\hat{R}(S))=\operatorname{depth}(S)$.

2. $\operatorname{depth}(\hat{R}(S))=\nu(\hat{R}(S)(k))<\nu(\hat{R}(S)(k+1))$ or $\operatorname{depth}(\hat{R}(S))=$ $\nu(\hat{R}(S)(k))>\nu(\hat{R}(S)(k-1))$, for some $k \in \mathbb{Z}$.

By (12), also in this case $\operatorname{depth}(\hat{R}(S))=\operatorname{depth}(S)$.

Now it follows from (12) that (14) is valid.

Theorem 4. Let $L \in \mathbb{C}\left[z, E_{z}\right]$, ord $L=d$, and let

$$
R=r_{d-1}(z) E_{z}^{d-1}+\cdots+r_{1}(z) E_{z}+r_{0}(z) \in \mathbb{C}(z)\left[E_{z}\right]
$$

be a summing operator for $L$. Denote by $V$ the set of all the poles of $r_{0}(z), r_{1}(z), \ldots, r_{d-1}(z)$. Then there exist non-zero $f, g \in \mathbb{C}^{\mathbb{Z}}$ such that

(i) $L(f(k))=0$ for all $k \in \mathbb{Z}$,

(ii) $g(k)=r_{d-1}(k) f(k+d-1)+\cdots+r_{1}(k) f(k+1)+r_{0}(k) f(k)$ for all $k \in \mathbb{Z} \backslash V$, and 
(iii) the discrete Newton-Leibniz formula

$$
\sum_{k=v}^{w-1} f(k)=g(w)-g(v)
$$

is valid for all integers $v<w$.

Proof. Pick any non-zero $U_{1}, \ldots, U_{d} \in \mathbb{C}((\varepsilon))$, and using $\hat{L}$ find a sequence $S \in$ $\mathbb{C}((\varepsilon))^{\mathbb{Z}}$ such that $S(i)=U_{i}, i=1,2, \ldots, d$, and $\hat{L}(S)=0$. So there exists a nonzero sequence $S$ such that $\hat{L}(S)=0$. Write $f=\operatorname{bott}(S), g=\operatorname{bott}(\hat{R}(S))$. Then (iii) is valid by Proposition 3, and (i) is valid since $L$ has polynomial coefficients. Finally, for all $k \notin V$ we have $g(k)=\operatorname{bott}(\hat{R}(S))(k)=R(\operatorname{bott}(S))(k)=R(f)(k)$, so (ii) is valid.

\section{The Analytic Case}

In the rest of this paper we assume that the sequences under consideration are defined on an infinite interval $I$ of integers, where either $I=\mathbb{Z}$, or

$$
I=\mathbb{Z}_{\geq l}=\{k \in \mathbb{Z} \mid k \geq l\}, \quad l \in \mathbb{Z} .
$$

It is easy to see that Propositions $1-3$ remain valid if we consider sequences defined on $\mathbb{Z}_{\geq l}$, and define the operators $\mathbb{Z}$ and bott with respect to $\mathbb{Z}_{\geq l}$ instead of with respect to $\mathbb{Z}$.

Let $U$ be an open subset of $\mathbb{C}$ containing $I$, such that $z \in U \Rightarrow z+1 \in U$. Denote by $\mathcal{M}(U)$ the set of functions which are meromorphic on $U$. We associate with $\varphi \in \mathcal{M}(U)$ a sequence $\hat{\varphi} \in \mathbb{C}((\varepsilon))^{\mathbb{Z}}$ whose $k$-th element, $k \in I$, is a (formal) series obtained by expanding $\varphi(\varepsilon+k)$ into Laurent series at $\varepsilon=0$.

Proposition 5. Let $L \in \mathbb{C}\left[z, E_{z}\right]$, and let $\varphi \in \mathcal{M}(U)$ satisfy $L(\varphi)=0$ on $U$. Then $\hat{L}(\hat{\varphi})=0$ everywhere on $\mathbb{Z}$, the sequence $\hat{\varphi} \in \mathbb{C}((\varepsilon))^{\mathbb{Z}}$ is of bounded depth, and $\tilde{L}(\operatorname{bott}(\hat{\varphi}))=0$ everywhere on $\mathbb{Z}$, where $\tilde{L}=\operatorname{bott}(\hat{L})$.

Proof. This follows from the trivial fact that the Laurent series of the zero function has only zero coefficients, and from Proposition 1.

Corollary 6. If $a_{0}, a_{1}, \ldots, a_{d} \in \mathbb{C}[z]$ then $\operatorname{bott}(\hat{L})=a_{d}(k) E_{k}^{d}+\cdots+a_{1}(k) E_{k}+$ $a_{0}(k)$. If in addition $S \in \mathbb{C}((\varepsilon))^{\mathbb{Z}}$ is such that $\hat{L}(S)=0$, then $L(\operatorname{bott}(S))=0$. In particular, if $\varphi \in \mathcal{M}(U)$ is such that $L(\varphi)=0$ everywhere on $U$ except possibly on a set of isolated points, then $L(\operatorname{bott}(\varphi))=0$ everywhere on $\mathbb{Z}$.

Example 2. In Example 1 we used, in fact, $L=(z+1) E_{z}-z, U=\mathbb{C}, \varphi(z)=-\frac{1}{z}$, $\Lambda=\hat{L}, S=\hat{\varphi}$. 
Theorem 7. (On the bottom summation.) Let $L \in \mathbb{C}\left[z, E_{z}\right]$, and let $R \in$ $\mathbb{C}(z)\left[E_{z}\right]$ be a summing operator for $L$. Let $\varphi \in \mathcal{M}(U)$ satisfy $L(\varphi)=0$ on $U$, and let $\psi=R(\varphi)$. Then the bottom summation formula

$$
\sum_{k=v}^{w-1} \operatorname{bott}(\hat{\varphi})(k)=\operatorname{bott}(\hat{\psi})(w)-\operatorname{bott}(\hat{\psi})(v)
$$

is valid for any $v<w, v, w \in I$. In particular, if $\varphi$ has no pole in $\mathbb{Z}$ (i.e., $\operatorname{depth}(\hat{\varphi})=0)$, then the function $\psi=R(\varphi) \in \mathcal{M}(U)$ has no pole in $\mathbb{Z}$, and the discrete Newton-Leibniz formula

$$
\sum_{k=v}^{w-1} \varphi(k)=\psi(w)-\psi(v)
$$

is valid for any $v<w, v, w \in I$.

Proof. The statement follows from Propositions 5, 3 .

Consider some known examples in the context of Theorem 7.

Example 3. The function $\varphi(z)=z \Gamma(z+1)$ satisfies the equation $L(y)=0$ where $L=z E_{z}-(z+1)^{2}$. We have $R=\frac{1}{z}$, ord $R=0$, and $\psi(z)=R(\varphi)(z)=\Gamma(z+1)$. Evidently $\varphi(z)$ has finite values when $z=0,1, \ldots$, and has simple poles when $z=-1,-2, \ldots$. If we consider $I=\mathbb{Z}$ then $\operatorname{depth}(\hat{\varphi})=\operatorname{depth}(\hat{\psi})=-1$ and

$$
\begin{aligned}
& \operatorname{bott}(\hat{\varphi})(k)= \begin{cases}\frac{(-1)^{k+1} k}{(-k-1) !}, & \text { if } k<0, \\
0, & \text { if } k \geq 0,\end{cases} \\
& \operatorname{bott}(\hat{\psi})(k)= \begin{cases}\frac{(-1)^{k+1}}{(-k-1) !}, & \text { if } k<0, \\
0, & \text { if } k \geq 0 .\end{cases}
\end{aligned}
$$

As a consequence of (15) we have

$$
\sum_{k=v}^{w-1} \frac{(-1)^{k} k}{(-k-1) !}=\frac{(-1)^{w}}{(-w-1) !}-\frac{(-1)^{v}}{(-v-1) !}
$$

for any $v<w \leq 0$, or equivalently

$$
\sum_{k=v}^{w-1} \frac{(-1)^{k} k}{(k-1) !}=\frac{(-1)^{w+1}}{(w-2) !}-\frac{(-1)^{v+1}}{(v-2) !}
$$

for any $1 \leq v<w$.

If $I=\overline{\mathbb{Z}}_{\geq 0}$ then $\operatorname{depth}(\hat{\varphi})=\operatorname{depth}(\hat{\psi})=0$, and by (16) we have $\sum_{k=v}^{w-1} k \Gamma(k+$ $1)=\Gamma(w+1)-\Gamma(v+1)$ for any $0 \leq v<w$ or, equivalently, $\sum_{k=v}^{w-1} k \cdot k !=w !-v$ !. 
Example 4. The rational function $\varphi(z)=\frac{1}{z(z+1)}$ satisfies the equation $L(y)=0$ where $L=(z+2) E_{z}-z$. We have $R=-z-1$, and $\psi(z)=R(\varphi)(z)=-\frac{1}{z}$. If we consider $I=\mathbb{Z}$ then $\operatorname{depth}(\hat{\varphi})=\operatorname{depth}(\hat{\psi})=-1$ and

$$
\begin{gathered}
\operatorname{bott}(\hat{\varphi})(k)=\delta_{0, k}-\delta_{-1, k}, \\
\operatorname{bott}(\hat{\psi})(k)=-\delta_{0, k},
\end{gathered}
$$

where $\delta$ is the Kronecker delta. A simple direct check shows that (15) is valid.

If $I=\mathbb{Z}_{\geq 1}$ then $\operatorname{depth}(\hat{\varphi})=\operatorname{depth}(\hat{\psi})=0$, and by (16) we have $\sum_{k=v}^{w-1} \frac{1}{k(k+1)}=-\frac{1}{w}+\frac{1}{v}$ for any $0 \leq v<w$.

The following example demonstrates a conflict between combinatorial and analytic definitions of the symbol $\left(\begin{array}{l}p \\ q\end{array}\right)$.

Example 5. Consider the hypergeometric sequence

$$
t(k)=\frac{\left(\begin{array}{c}
2 k-3 \\
k
\end{array}\right)}{4^{k}}
$$

which satisfies the equation $2(k+1)(k-2) t(k+1)-(2 k-1)(k-1)=0$. It has been noticed in [5] that even though Gosper's algorithm succeeds on this sequence, producing $R(k)=\frac{2 k(k+1)}{k-2}$, and $t(k)$ is defined for all $k \in \mathbb{Z}$, the discrete Newton-Leibniz formula

$$
\sum_{k=0}^{w-1} t(k)=R(w) t(w)-R(0) t(0)=\frac{2 w(w+1)\left(\begin{array}{c}
2 w-3 \\
w
\end{array}\right)}{(w-2) 4^{w}}
$$

is not correct. If we assume that the value of $\left(\begin{array}{c}2 k-3 \\ k\end{array}\right)$ is 1 when $k=0$ and -1 when $k=1$ (as is common practice in combinatorics) then the expression on the right gives the true value of the sum only at $w=1$. However, assume that the value of $\left(\begin{array}{c}2 k-3 \\ k\end{array}\right)$ is defined as

$$
\lim _{z \rightarrow k} \frac{\Gamma(2 z-2)}{\Gamma(z+1) \Gamma(z-2)} .
$$

This limit exists for all $k \in \mathbb{Z}$, but

$$
\lim _{z \rightarrow 0} \frac{\Gamma(2 z-2)}{\Gamma(z+1) \Gamma(z-2)}=\frac{1}{2} \neq 1
$$

and

$$
\lim _{z \rightarrow 1} \frac{\Gamma(2 z-2)}{\Gamma(z+1) \Gamma(z-2)}=-\frac{1}{2} \neq-1 .
$$

Set

$$
\varphi(z)=\frac{\Gamma(2 z-2)}{\Gamma(z+1) \Gamma(z-2) 4^{z}}
$$


and

$$
\psi(z)=\frac{2 z(z+1)}{z-2} \varphi(z) .
$$

Then formula (16) gives the correct result

$$
\sum_{k=0}^{w-1} \frac{\Gamma(2 k-2)}{\Gamma(k+1) \Gamma(k-2) 4^{k}}=\frac{2 w(w+1) \Gamma(2 w-2)}{(w-2) \Gamma(w+1) \Gamma(w-2) 4^{w}}
$$

for all $w \geq 1$, provided that the values of the summand and of the right-hand side are defined by taking appropriate limits.

Note that if $\alpha k_{0}+\beta$ is a non-positive integer, then we can often avoid a direct computation of limits using the asymptotic equality

$$
\Gamma(\alpha z+\beta) \sim \frac{(-1)^{\alpha k_{0}+\beta}}{\left(-\alpha k_{0}-\beta\right) ! \cdot \alpha \cdot\left(z-k_{0}\right)}, \quad z \rightarrow k_{0},
$$

instead. If $\alpha \neq 0$ and $-\frac{\beta}{\alpha}$ is an integer $\gamma$, then $\Gamma(\alpha z+\beta)$ has integer poles at $\gamma, \gamma-1, \ldots$ if $\alpha>0$ and $\gamma, \gamma+1, \ldots$ if $\alpha<0$.

The following example is related to the case ord $L>1$.

Example 6. For the operator $L=(z-3)(z-2)(z+1) E_{z}^{2}-(z-3)\left(z^{2}-2 z-\right.$ 1) $E_{z}-(z-2)^{2}$ there exists the summing operator

$$
R=z E_{z}+\frac{1}{z-3}
$$

([5]). By [6] the equation $L(y)=0$ has solutions holomorphic at the half-plane $\operatorname{Re} z>2$. Denote by $\varphi(z)$ an arbitrary solution of this kind. By Theorem 7 , formula (16) must be correct for the case $I=\mathbb{Z}_{\geq 3}$ in spite of the fact that one of the coefficients of $R$ has a pole at $z=3$. This implies that $\varphi(z)$ vanishes at $z=3$. This can be easily confirmed by the substitution of $z=3$ into $L(\varphi)=0$, which results in $-\varphi(3)=0$. The algorithm from [4] yields $\varphi(z)=(\varphi(4)+4 \varphi(5))(z-$ $3)+O\left((z-3)^{2}\right)$, and formula (16) gives the correct result for $3 \leq v<w$.

\section{Conclusion}

Indiscriminate application of the discrete Newton-Leibniz formula to the output of Gosper's algorithm or of the Accurate Summation algorithm in order to compute a definite sum can lead to incorrect results. This can be observed in many implementations of these algorithms in computer algebra systems.

In the present paper it is shown, in particular, that such undesirable phenomena cannot occur if the elements of the sequence under summation are the values $\varphi(k), k \in \mathbb{Z}$, of an analytic function $\varphi(z)$, which satisfies (in the complex plane $\mathbb{C}$ ) the same difference equation with polynomial coefficients as does the original sequence (at integer points). 
A practical consequence of this results is as follows. If the conditions formulated above are satisfied, then a computer-algebra-system user can be sure that the obtained sum was computed correctly.

On the more theoretical side, if $\varphi(z)$ mentioned above has some poles at integer points, then nevertheless one can find the sum of a sequence which, however, is not the sequence of values of $\varphi(k), k \in \mathbb{Z}$, but is associated with $\varphi(z)$ in a natural way. This can yield an interesting (and, probably, unexpected) identity. We call this sequence associated with $\varphi(z)$, the bottom of $\varphi(z)$. If $\varphi(z)$ is defined for all $z \in \mathbb{Z}$ then its bottom coincides with the sequence $\varphi(k), k \in \mathbb{Z}$.

\section{References}

1. S. A. Abramov, On the summation of $P$-recursive sequences. ISSAC'06 (Annual International Symposium on Symbolic and Algebraic Computation). Genova, Italia; 9-12 July 2005; Proceedings ISSAC'06, 17-22.

2. S. A. Abramov, M. A. Barkatou, M. van Hoeij, Apparent singularities of linear difference equations with polynomial coefficients. AAECC (Applicable Algebra in Engineering, Communication and Computing) 17 (2006), 117-133.

3. S. A. Abramov, M. van Hoeij, Integration of solutions of linear functional equations. Integral Transforms and Special Functions 8 (1999), 3-12.

4. S. A. Abramov, M. van Hoeij, Set of poles of solutions of linear difference equations with polynomial coefficients. J. Comput. Math. Math. Phys. 43 (2003), 57-62. (Translated from Zhurnal Vychislitel'noi Matematiki i Matematicheskoi Fiziki $\mathbf{4 3}$ (2003), 60-65.)

5. S. A. Abramov, M. Petkovšek, Gosper's Algorithm, Accurate Summation, and the discrete Newton-Leibniz formula. ISSAC'05 (Annual International Symposium on Symbolic and Algebraic Computation). Beijing, China; 24-27 July 2005; Proceedings ISSAC'05, 5-12.

6. M. A. Barkatou, Contribution à l'étude des équations différentielles et aux différences dans le champ complexe. PhD Thesis, INPG Grenoble, France, 1989.

7. M. van Hoeij, Finite singularities and hypergeometric solutions of linear recurrence equations. J. Pure Appl. Algebra 139 (1999), 109-131.

8. R. W. Gosper, Jr., Decision procedure for indefinite hypergeometric summation. Proc. Natl. Acad. Sci. USA $\mathbf{7 5}$ (1978), 40-42.

This article was processed using the $\mathrm{LT}_{\mathrm{E}} \mathrm{X}$ macro package with LLNCS style 